\title{
Analysis of a $38 \mathrm{GHz}$ Band Antenna for $5 \mathrm{G}$ wireless communication using Wave Concept Iterative Procedure (WCIP) method
}

\author{
Doae EL HADRI ${ }^{1}$, Asmaa ZUGARI ${ }^{2}$ and Alia ZAKRITI ${ }^{1}$ \\ doae93@gmail.com, asmaa.zugari@gmail.com, aazakriti@hotmail.fr \\ ${ }^{1}$ Advanced Science and Technology Group, Physics Department, ENSA \\ Abdelmalek Essaadi University,Tetuan, Morocco \\ ${ }^{2}$ Laboratory Systems of Information and Telecommunications, FS \\ Abdelmalek Essaadi University,Tetuan, Morocco
}

\begin{abstract}
The analysis of mm-Wave patch antenna for future fifth generation (5G) wireless communications is presented in this paper. The antenna is mounted on a compact Rogers substrate having dimensions $6.336 \times 6.4 \times 0.254 \mathrm{~mm}^{3}$ with relative permittivity (Er) 2.2. The proposed antenna operates at a $38 \mathrm{GHz}$ band. It is modelled by the Wave Concept Iterative Procedure (WCIP) method. The iterative procedure applies the fast modal transform (FMT) to change the analysis between spatial and spectral domains. In order to validate the present results we used the commercially available software CST MICROWAVE STUDIO. The numerical results show a good agreement compared with the CST simulation data, which illustrates the efficiency and correctness of our WCIP algorithm.
\end{abstract}

Keywords: 5G wireless communication, Antenna, CST, Wave Concept Iterative Procedure, MATLAB.

\section{Introduction}

The successful development of mobile wireless communications is demonstrated by a rapid rhythm of technology innovation. This growth has seen many generations from 1G, 2G, 3G, 4G and $5 \mathrm{G}$. Each of these generations have various wireless technologies, data rates, modulation techniques, capacities and features compared to the other.

In initial 1980's the first generation (1G) mobile system was announced and supports only analog voice. The second generation (2G) mobile communication system debuted in 1991, it uses digital signals for voice transmission and has speed of $64 \mathrm{kbps} .2 .5 \mathrm{G}$ system provides data rate up to $144 \mathrm{kbps}$ [1]. The $3 \mathrm{G}$ system first launched in 2001 , it operates at a range of $2100 \mathrm{MHz}$ and has a bandwidth of $15-20 \mathrm{MHz}$. From $2 \mathrm{G}$ to $3 \mathrm{G}$ the wireless mobile network has been transformed from a pure telephony system to a network that can transport rich multimedia contents [2]. 
Fourth generation $(4 \mathrm{G})$ wireless networks can support data rates of up to $1 \mathrm{~GB} / \mathrm{s}$ for low mobility, and up to $100 \mathrm{Mb} / \mathrm{s}$ for high mobility. The technologies driving 4G growth are LTE and WiMAX.

However, 4G still cannot accommodate some challenges like spectrum crisis, high energy consumption, poor coverage, bad interconnectivity, poor Quality of Service (QoS) and flexibility. To address all these demands $5 \mathrm{G}$ wireless system are expected to be deployed in the future by 2020 [3].

The fifth-generation $(5 \mathrm{G})$ cellular systems is more than an evolution of mobile broadband. It will be a key enabler of the future digital world, it is an opportunity to launch brand new internet services such as internet of things(IoT), mission critical services, high definition video stream, bitpipe communications at Gbps, tactile internet [4], rural access networks and autonomous car [5].

In order to meet the growing demand for more connected objects and higher data rates, the fifth generation of mobile network (5G) will be deployed. Compared to current mobile communication systems, new five technologies would be adopted like, device-centric architectures, millimeter wave, massive multiple-in multiple-out (MIMO), smarter devices, and native support for machine-to-machine (M2M) communications. These technologies may provide $10-100 \times$ higher user data rate, $1000 \times$ higher mobile data volume per area, 10-100× higher number of connected devices, $10 \times$ longer battery lifetime, and $5 \times$ reduced end-to-end latency [6].

The realization of these high level technologies brings about new challenges for the physical infrastructure designers which undoubtedly include the antenna and microwave engineers. From the electromagnetic wave perspective, due to this unprecedented growth of mobile data demand, the very limited spectrum resources available in the sub- $6 \mathrm{GHz}$ spectrum no longer satisfy the system needs. Consequently, the use of the vast amount of underutilized spectrum in the $6-300 \mathrm{GHz}$ range for next-generation commercial wireless systems has recently garnered a great amount of research interest [7]. Accordingly, the mm-Wave spectrum was allocated for $5 \mathrm{G}$ communications [8].

The antenna is the most key components of wireless systems as it intensely affects the total receiver sensitivity, thus transceiver designs and choices of digital modulation schemes and the link budget [9]. It play an essential role in the field of wireless communication. It consists of slot antennas, patch antennas.... [10].

This paper analysed an antenna design that is inspired from recent literature [11] by modification of parameters, for mm-wave $5 \mathrm{G}$ applications operating in the $38-\mathrm{GHz}$ using Wave Concept Iterative Procedure (WCIP) method. The advantage of this method remains in its ease of use due to the absence of test functions, its fast computation time, mainly due to the systematic use of Fast Mode Transform (FMT) and surface mesh use. In addition, there is no matrix inversion was required and the convergence was insured independently of the circuit complexity.The numerical results obtained are validated using the commercially available software CST MICROWAVE STUDIO. 


\section{Wave Concept Iterative Procedure}

\subsection{Formulation}

The Wave Concept Iterative Procedure is an integral method based on wave concept [12], the waves are defined conveniently as a combination of the transverse electric field and current density, normalized in terms of root of impedance. These waves, incident A and reflected B, on the discontinuity surface $(\Omega)$ are written by the following relationships (1) [13]:

$$
\left\{\begin{array}{l}
\mathrm{A}_{\mathrm{i}}=\frac{1}{2 \sqrt{\mathrm{Z}_{0 \mathrm{i}}}}\left(\mathrm{E}_{\mathrm{Ti}}+\mathrm{Z}_{0 \mathrm{i}} \mathrm{J}_{\mathrm{Ti}}\right) \\
\mathrm{B}_{\mathrm{i}}=\frac{1}{2 \sqrt{\mathrm{Z}_{0 \mathrm{i}}}}\left(\mathrm{E}_{\mathrm{Ti}}-\mathrm{Z}_{0 \mathrm{i}} \mathrm{J}_{\mathrm{Ti}}\right)
\end{array}\right.
$$

Where $\mathrm{Z}_{0 \mathrm{i}}$ is the characteristic impedance of the medium $\mathrm{i}(\mathrm{i}=1,2)$.

The transverse waves $\mathrm{Ai}$ and $\mathrm{Bi}$ can be used to calculate the tangential electric fields and the current intensity of the interface as (2):

$$
\left\{\begin{array}{l}
\overrightarrow{\mathrm{E}_{1}}=\sqrt{\mathrm{Z}_{0 \mathrm{i}}}\left(\overrightarrow{\mathrm{A}_{1}}+\overrightarrow{\mathrm{B}_{1}}\right) \\
\overrightarrow{\mathrm{J}_{1}}=\frac{1}{\sqrt{\mathrm{Z}_{0 \mathrm{i}}}}\left(\overrightarrow{\mathrm{A}_{1}}-\overrightarrow{\mathrm{B}_{1}}\right)
\end{array}\right.
$$

The analytical expression of the iterative process involves a system of two equations: one written in the spatial domain (3), characterizing the boundary conditions at the interface of the structure through the operator $\widehat{S}$; the other expressed in the modal domain (4), describing the upper and lower media through the operator $\widehat{\Gamma}[14]$. The iterative process is defined by:

$$
\begin{cases}\vec{A}=\hat{S} \vec{B}+\vec{A}_{0} & \text { Spatial domain } \\ \widetilde{B}=\hat{\Gamma} \check{A} & \text { Spectral domain }\end{cases}
$$

$A_{0}$ is the incident wave generated by the source

The connection between the spatial domain and spectral domain is carried out via the Fast Modal Transform (FMT) (Figure 1) and its inverse $\left(\mathrm{FMT}^{-1}\right)$ [15]. In each step, the incident and reflected waves are determined. 


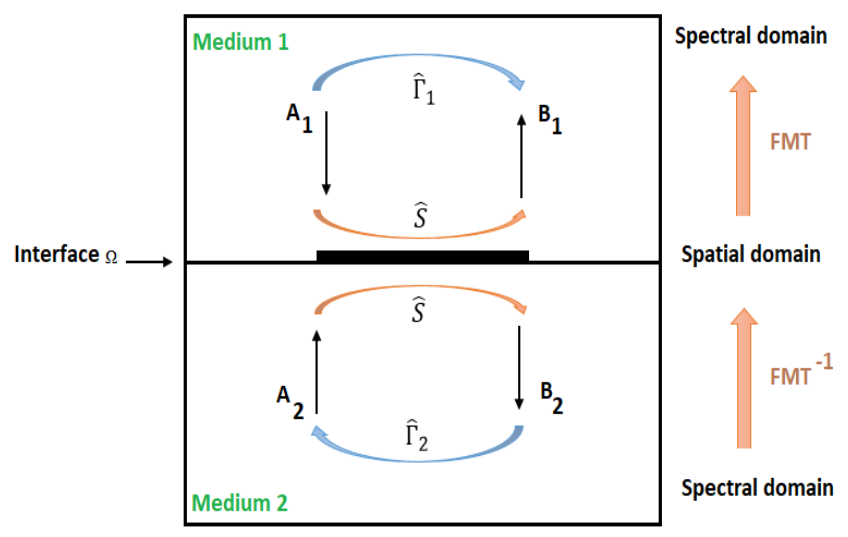

Fig. 1. Wave Concept Iterative Procedure process.

The scattering matrix of the general structure is show in equation (5) [16], it build by the physical nature of the material: if it is a source, a dielectric or a metal.

$$
[\widehat{S}]=\left[\begin{array}{cc}
-H_{m}-\frac{n^{2}-1}{n^{2}+1} H_{d}+\frac{-1+n_{1}-n_{2}}{1+n_{1}+n_{2}} H_{S} & \frac{2 n}{n^{2}+1} H_{d}+\frac{n_{3}}{1+n_{1}+n_{2}} H_{S} \\
\frac{2 n}{n^{2}+1} H_{d}+\frac{n_{3}}{1+n_{1}+n_{2}} H_{S} & -H_{m}+\frac{n^{2}-1}{n^{2}+1} H_{d}+\frac{-1-n_{1}+n_{2}}{1+n_{1}+n_{2}} H_{S}
\end{array}\right]
$$

Where : $H_{i}=\left\{\begin{array}{rr}1 & \text { on the i domain } \\ 0 & \text { other domain }\end{array} \quad(i=\right.$ Metal, Dielectric, Source $)$

$$
\mathrm{n}=\sqrt{\frac{\mathrm{Z}_{01}}{\mathrm{Z}_{02}}}, \mathrm{n}_{1}=\frac{\mathrm{Z}_{0}}{\mathrm{Z}_{01}}, \mathrm{n}_{2}=\frac{\mathrm{Z}_{0}}{\mathrm{Z}_{02}}, \mathrm{n}_{3}=\frac{2 \mathrm{Z}_{0}}{\sqrt{\mathrm{Z}_{01}} \sqrt{\mathrm{Z}_{02}}} \text { and }\left[\begin{array}{l}
\mathrm{A}_{01} \\
\mathrm{~A}_{02}
\end{array}\right]=\frac{1}{1+\mathrm{n}_{1}+\mathrm{n}_{2}}\left[\begin{array}{l}
\frac{\mathrm{E}_{0}}{\sqrt{\mathrm{Z}_{01}}} \mathrm{H}_{\mathrm{S}} \\
\frac{\mathrm{E}_{0}}{\sqrt{\mathrm{Z}_{02}}} \mathrm{H}_{\mathrm{S}}
\end{array}\right]
$$

The expression of reflection operator in the modal domain is given by equation (6) [17]:

$$
\widehat{\Gamma}=\sum_{\mathrm{m}, \mathrm{n}}\left|\mathrm{f}_{\mathrm{mn}}^{\mathrm{TE}}\right\rangle \Gamma_{\mathrm{mn}}^{\mathrm{TE}}\left\langle\mathrm{f}_{\mathrm{mn}}^{\mathrm{TE}}\left|+\sum_{\mathrm{m}, \mathrm{n}}\right| \mathrm{f}_{\mathrm{mn}}^{\mathrm{TM}}\right\rangle \Gamma_{\mathrm{mn}}^{\mathrm{TM}}\left\langle\mathrm{f}_{\mathrm{mn}}^{\mathrm{TM}}\right|
$$

Where :

$$
\Gamma_{\mathrm{mn}}^{\alpha}=\frac{1-\mathrm{Z}_{0 \mathrm{i}} \mathrm{Y}_{\mathrm{mn}}^{\alpha}}{1+\mathrm{Z}_{0 \mathrm{i}} \mathrm{Y}_{\mathrm{mn}}^{\alpha}}
$$

$Y_{m n}^{\alpha}$ Can be calculated by equation (7) :

$$
\mathrm{Y}_{\mathrm{mn}}^{\mathrm{TE}}\left(\varepsilon_{\mathrm{r}}\right)=\frac{\gamma_{\mathrm{mn}}}{\mathrm{j} \omega \mu_{0}} \quad \text { et } \quad \mathrm{Y}_{\mathrm{mn}}^{\mathrm{TM}}\left(\varepsilon_{\mathrm{r}}\right)=\frac{\mathrm{j} \omega \varepsilon_{0} \varepsilon_{\mathrm{r}}}{\gamma_{\mathrm{mn}}}
$$


$\gamma_{m n}$ being the propagation constant of the medium $\mathrm{i}$ and it is given by (8) :

$$
\left(\gamma_{\mathrm{mn}}^{2}\right)=\left(\frac{\mathrm{m} \pi}{\mathrm{a}}\right)^{2}+\left(\frac{\mathrm{n} \pi}{\mathrm{b}}\right)^{2}-\mathrm{K}_{0}^{2}\left(\varepsilon_{\mathrm{r}}\right)_{\mathrm{i}}
$$

\section{Antenna Design}

Figure 2 shows the different antenna structures, which are investigated during the simulation studies, and their resonance frequencies of each case are displayed :

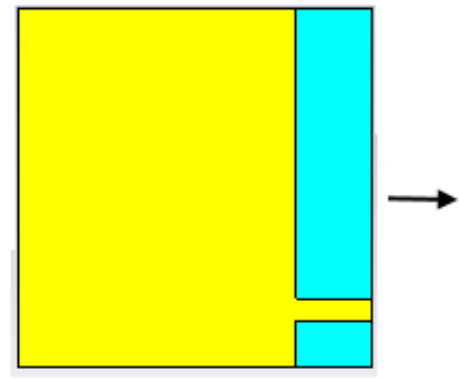

$f_{r}=39.135 \mathrm{GHz}$

Case (1)

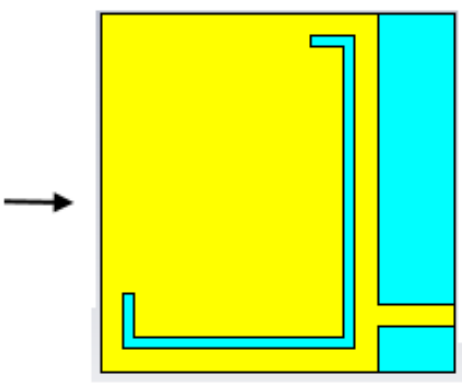

$f_{r}=35.85 \mathrm{GHz}$

Case (2)

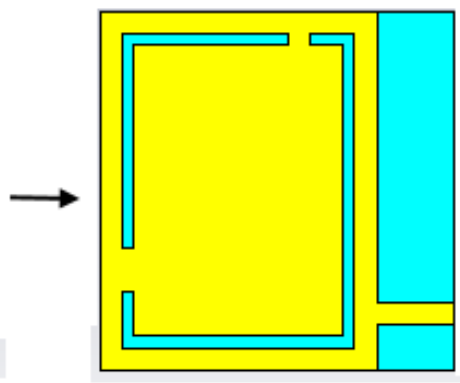

$f_{r}=38 \mathrm{GHz}$

Case (3)

Fig. 2. Design evolution of the proposed antenna

After optimizing the antenna parameters, the geometry of the proposed antenna for future $5 \mathrm{G}$ wireless communications is shown in Figure 3. The patch is designed using conducting material with slots. The antenna is printed on a Rogers substrate with dielectric constant 2.2 and thickness $\mathrm{h}=0.254 \mathrm{~mm}$. The ground plane is made up of same conducting material as the patch.

The discretization process applied by the WCIP interface shown in Figure 4. The dimensions of the interface are $6.336 \times 6.4 \mathrm{~mm}^{2}$.
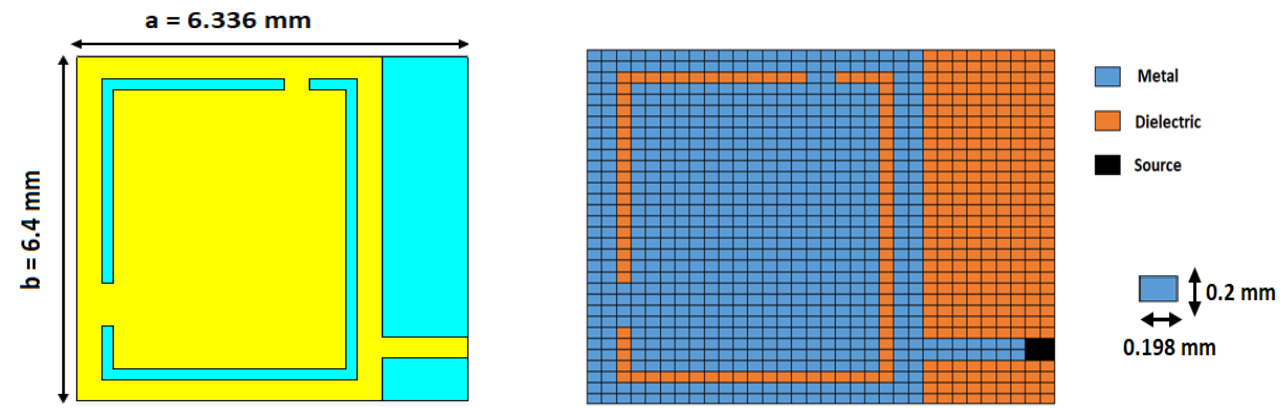

Fig. 3. Geometry of the proposed antenna.

Fig. 4. Discretization of $5 \mathrm{G}$ antenna in the WCIP interface. 


\section{Numerical results}

To ensure that the method WCIP has achieved convergence, a study is conducted at a frequency point at $36 \mathrm{GHz}$. The result presented in Figure $\mathbf{5}$ shows the convergence of the $\mathrm{S}$ parameter module as a function of the number of iterations. This study is recommended to predefine an estimate of the number of iterations required by the method to achieve the result at each frequency point.

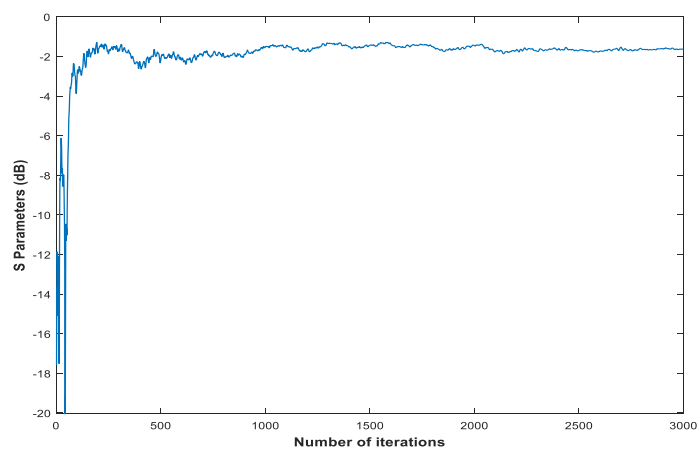

Fig. 5. Convergence of the $\mathrm{S}$ parameter at $36 \mathrm{GHz}$

The distribution of the electric field and current density, which is shown in Figure 6, conforms to the boundary conditions of the circuit interface.
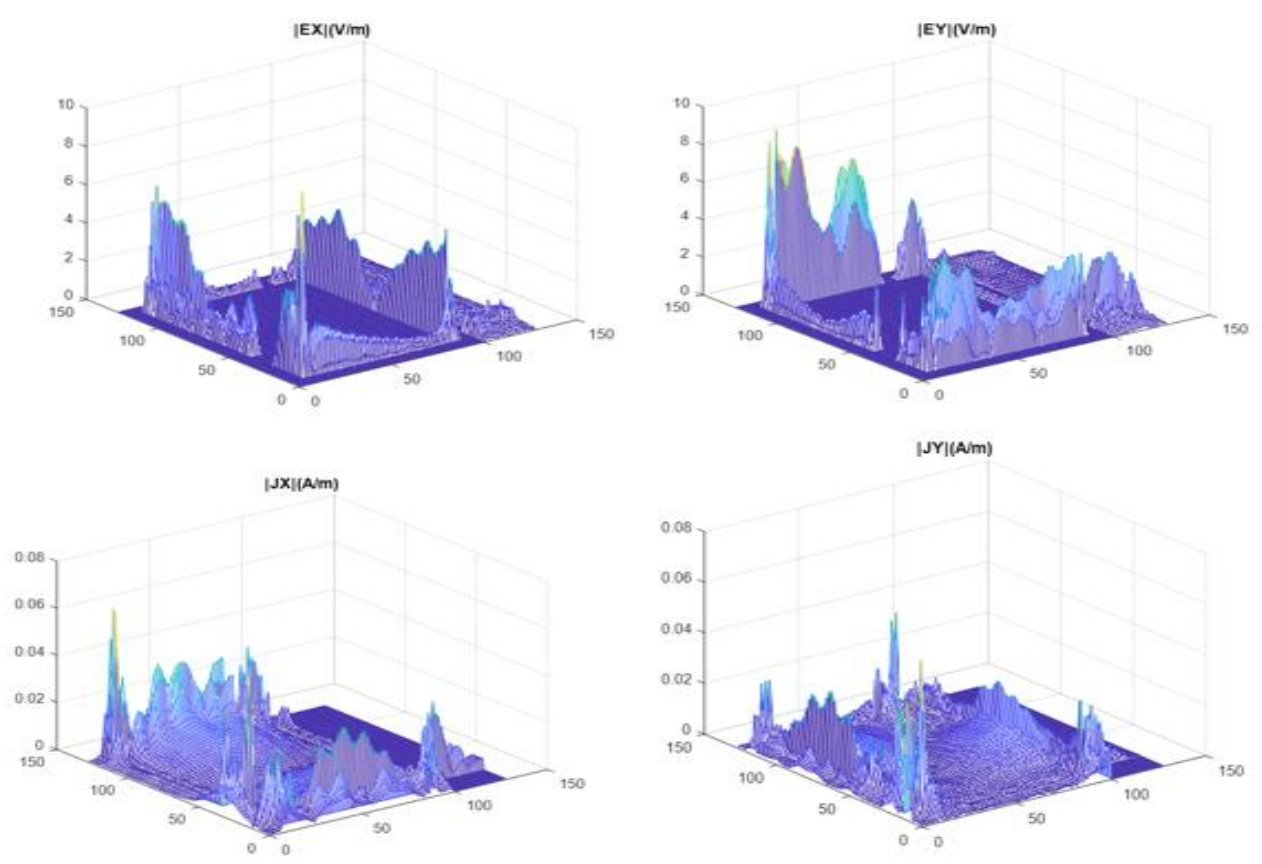

Fig. 6. Distribution of the electric field and current density 
The calculated S parameters of the antenna with WCIP method show a good agreement with the CST results as presented in Figure 7. The resonance frequency is $38 \mathrm{GHz}$. The bandwidth at $-10 \mathrm{~dB}$ is of order of $2 \mathrm{GHz}$ (WCIP results). The phase of the reflection coefficient is presented in the Figure 8.
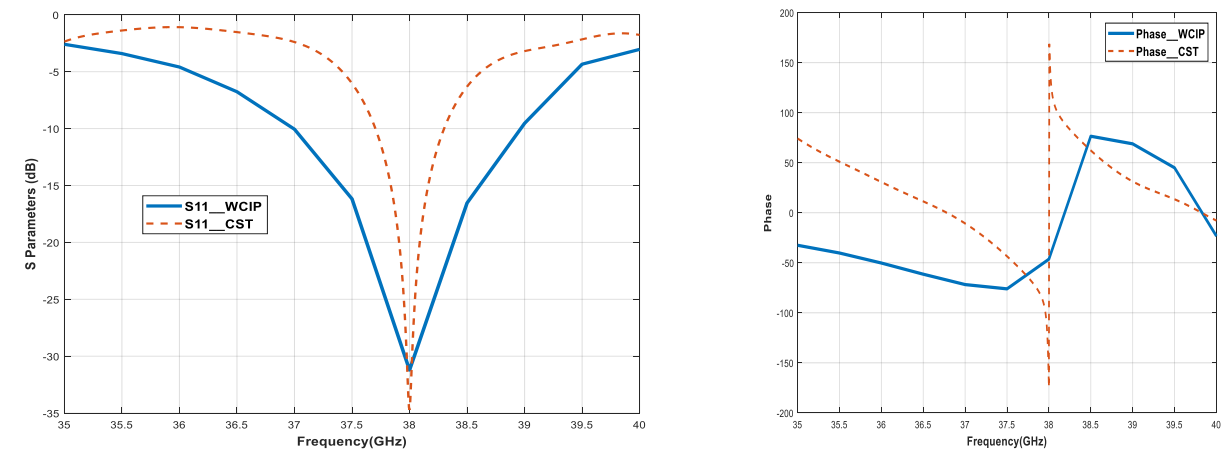

Fig. 7. The reflection coefficients (S11) for proposed antenna

Fig. 8. The reflection coefficient phase

The figure below (Figure 9) shows the VSWR of the proposed antenna, the value of this parameter is compared using WCIP and CST in Table $\mathbf{1}$ for the resonance frequency $(38 \mathrm{GHz})$.

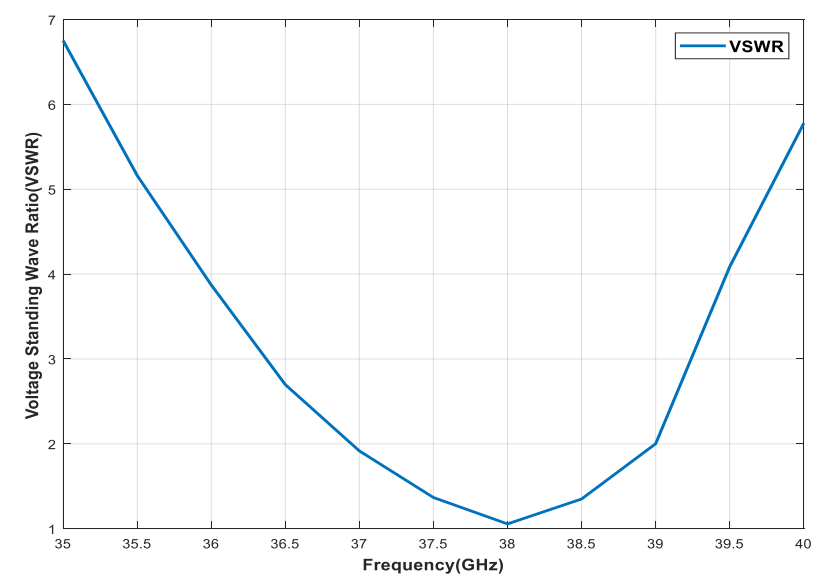

Fig. 9. The Voltage Standing Wave Ratio (VSWR)

Table 1. comparative table of the VSWR parameter at $38 \mathrm{GHz}$ frequency

\begin{tabular}{lll}
\hline Parameter & WCIP & CST \\
\hline VSWR & 1.057 & 1.037 \\
\hline
\end{tabular}

The gain of proposed $5 \mathrm{G}$ antenna for wireless communication at $38 \mathrm{GHz}$ was $6.95 \mathrm{dBi}$. 


\section{Conclusion}

In this paper, the analysis of $5 \mathrm{G}$ antenna for wireles communication using an iterative method, is carried out and discussed. The $S_{11}$ parameters, the phases of the reflection coefficient, and VSWR for the antenna are determined. The resonance frequency is in good agreement between CST and WCIP simulation.

\section{Acknowledgments:}

The work is carried out with the support of the CNRST within the framework of the Research Excellence Scholarship Programme.

\section{References}

[1] G. R. Patil and P. S.Wankhade, “5G WIRELESS TECHNOLOGY,” Int. J. Comput. Sci. Mob. Comput., vol. 3, no. 10, pp. 203-207, 2014.

[2] B. V Chaithanya and S. N. Shettar, "Smart Antenna Technology for 5g Wireless Communications," J. Android, IOS Dev. Test., vol. 2, no. 1, pp. 1-12, 2017.

[3] N. K. Reddy, A. Hazra, and V. Sukhadeve, "A Compact Elliptical Microstrip Patch Antenna for Future 5G Mobile Wireless Communication,” Trans. Eng. Appl. Sci., pp. 1-4, 2017.

[4] T. S. Rappaport, Y. Xing, G. R. Maccartney, A. F. Molisch, E. Mellios, and J. Zhang, "Overview of Millimeter Wave Communications for Fifth-Generation (5G) Wireless Networks-With a Focus on Propagation Models,” IEEE Trans. Antennas Propag., vol. 65, no. 12, pp. 6213-6230, 2017.

[5] A. C. S. Jr, “Antenna Development for 5G Networks,” IEEE, pp. 2-6, 2017.

[6] P. Zhang, J. Lu, Y. Wang, and Q. Wang, "Cooperative localization in 5G networks : A survey," ICT Express, vol. 3, no. 1, pp. 27-32, 2017.

[7] W. Hong et al., "Multi - Beam Antenna Technologies for 5G Wireless Communications," IEEE Trans. Antennas Propag. Submitt., no. c, 2017.

[8] W. El-halwagy, R. Mirzavand, M. Jordan, H. Masum, and M. Pedram, "Investigation of Wideband Substrate-Integrated Vertically-Polarized Electric Dipole Antenna and Arrays for mmWave 5G Mobile Devices," IEEE Access, no. December, 2017.

[9] M. A. Matin, "Review on Millimeter Wave Antennas- Potential Candidate for 5G Enabled Applications,” Adv. Electromagn., vol. 5, no. 3, 2016.

[10] R. A. Panda, N. Patnaik, C. Dash, and B. Kumari, "Dual Band Modified E-Shaped Microstrip Patch Antenna for 5g Application and Space Communication,” Int. J. Res. Appl. Sci. Eng. Technol., no. September, 2017.

[11] M. El Shorbagyl, R. M. Shubair, M. I. Aihajri, and N. K. Mallat, "On the Design of MillimetreWave Antennas for 5G," IEEE, pp. 7-10, 2016.

[12] N. Raveu, G. Prigent, T. Callegari, and H. Baudrand, "WCIP APPLIED TO ACTIVE PLASMA CIRCUITS,” Prog. Electromagn. Res. Lett., vol. 21, no. January, pp. 89-98, 2011

[13] G. Fontgalland, P. I. L. Ferreira, T.-P. Vuong, N. Raveu, and H. Baudrand, "Analysis of asymmetric PBG ground planes for wireless communications,” IEEE Xplore, pp. 352-355, 2005.

[14] T. Ziar, S. Mellal, F. Hichem, M. Zaabat, and C. Azizi, "The study of the miniaturisation effect on the characteristics of patch antenna using the WCIP method," J. New Technol. Mater., vol. 4, pp. 53-57, 2014.

[15] A. I. Alhzzoury, N. Raveu, O. Pigaglio, H. Baudrand, and K. Al-Abdullah, "WCIP APPLIED TO SUBSTRATE INTEGRATED,” Prog. Electromagn. Res., vol. 33, no. August, pp. 171-184, 2012. 
[16] J. Wu-sheng, L. Quan-zhen, and Y. Fan, "Analysis of H-shaped patch antenna by Wave concept iterative procedure ( WCIP ), " IEEE, pp. 797-800, 2010.

[17] M. Tellache, Y. Lamhene, B. Haraoubia, and H. Baudrand, "AN NUMERICAL METHOD BASED ITERATIVE PROCESS TO CHARACTERIZE MICROWAVE PLANAR CIRCUITS," Int. J. Comput., vol. 7, no. 3, pp. 86-94, 2008. 